\title{
Automatic dust-free wiper
}

\author{
Fenglan wang ${ }^{1, a}$, Xingyang Jia ${ }^{2, b}$, Bo Zhang ${ }^{3, ~ c}$, Bo Gao ${ }^{4, d}$ \\ ${ }^{4}$ Shenyang University, No.21, Wanghua South Street, Dadong District, Shenyang City, Liaoning \\ Province, China \\ aflwang1965@sina.com, ${ }^{\text {b22009198901@163.com, }{ }^{\mathrm{d}} 497601981 @ q q . c o m}$
}

Keywords: fully automatic blackboard eraser, dust-free, mechanical transmission.

\begin{abstract}
The traditional blackboard is to rely on artificial wiped the handwriting effort and produce dust, causing inconvenience to the teacher in class, also affect the quality of students listening lectures. Institutional innovation reform to solve these problems by this design, the basis of the principle of mechanical transmission, avoiding artificial effort also improve the students listening environment, replace the traditional blackboard whole process of automatic polishing off the writing on the blackboard and automatic vacuuming clean, environmental protection and the structure is simple, manufacture easily, promote efficiency of the teacher in class.
\end{abstract}

\section{Introduction}

Everyone was a student once, in the age of the students are also through such a process. In order to erase the blackboard writing, they have to use the traditional blackboard eraser. That has a lot of dust board which is really a nightmare every time. When I was a student, it is known to all that the writing of the eraser be removed by simple blackboard, but there was not produce ash clean up in time, although to erase the effect of the handwriting it left potential dangers to student's physical health after that This is inevitable at the time, not only so, and completely by human, it is also helpless to a student and teacher, this is not only time-consuming, affect the quality of students listening the lectures, and dust and frequent the same action also cause harm to people's physical for a long time, If there is a kind of both energy saving and no dust wiper, it would be greatly welcomed by teachers and students. But now, there is born a kind of the blackboard eraser is convenient, it is full automatic dust-free wiper.

Drawing1Automatic dust-free blackboard eraser mechanism motion diagram (front view)

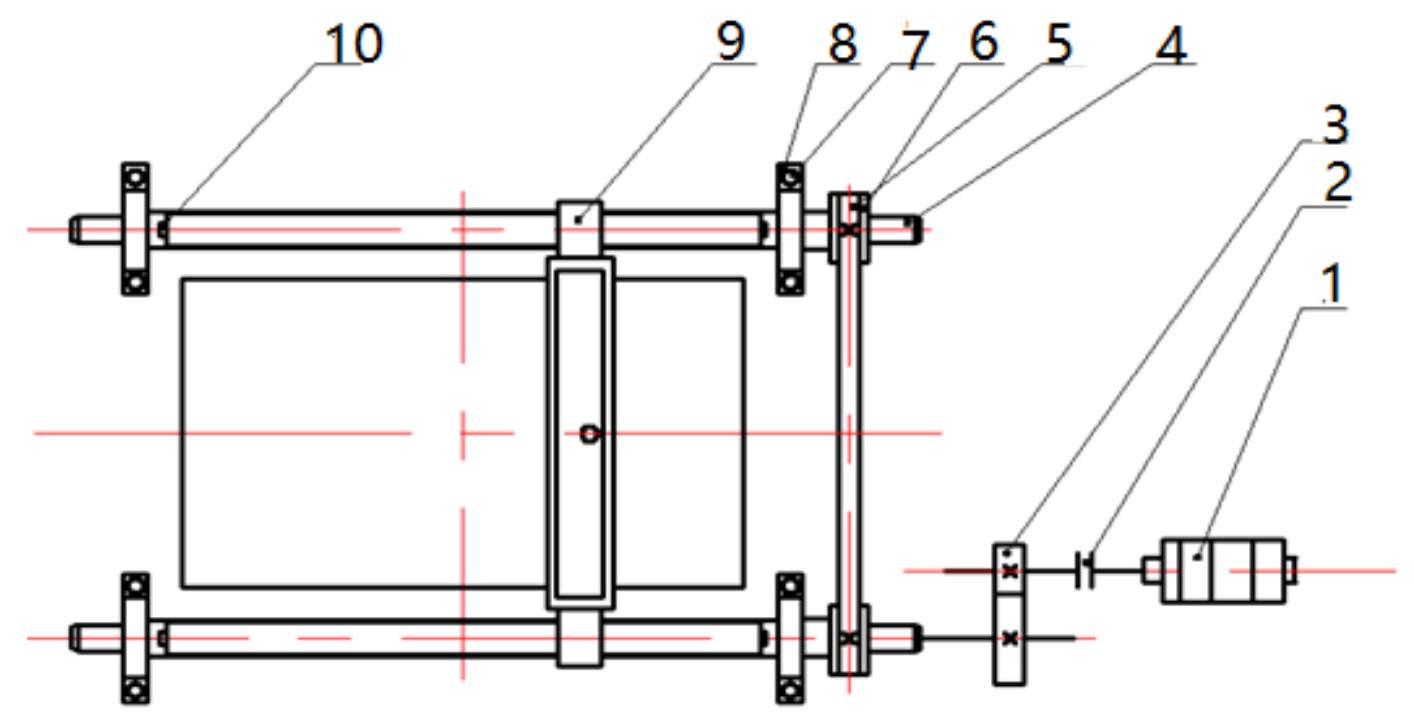

In the picture: 1- motor, 2 - coupling, 3 - reducer, 4 - screw, 5 - belt, 6 - pulley, 7 - screw, bracket of 8 -, 9 - an eraser, 10 - travel switch 
This design requires a power plant, meaning of transmission device, and the waste collection and stop sensor, so this design is composed of PLC control circuit and sensor, main control board polishing the movement back and forth. Blackboard eraser work moving at a constant speed, do not cause dangerous, because of unstable factors. When touching the blackboard end, it will stop automatically, this work is done. If due to too much quality problems or the board itself, we can press the control button, it will start again. After that, according to the circumstance the user may use it arbitrarily. We choose small three-phase asynchronous motor, reducer adopts level, this kind of choice can guarantee student safety, controlling of lead screw and nut mechanical mechanism is also a part of it. The two part work together to realize the blackboard polishing the mobile. As one of the characteristics of this kind of innovation, We should adopt covers the board cover polishing in the process of wiping covered ash, the use of grey hood greatly ensures that the wiping dust not in public in the process of polishing

Picture2 Down the motor, coupling, reducer, pulley, screw support two-dimensional chart view (left)

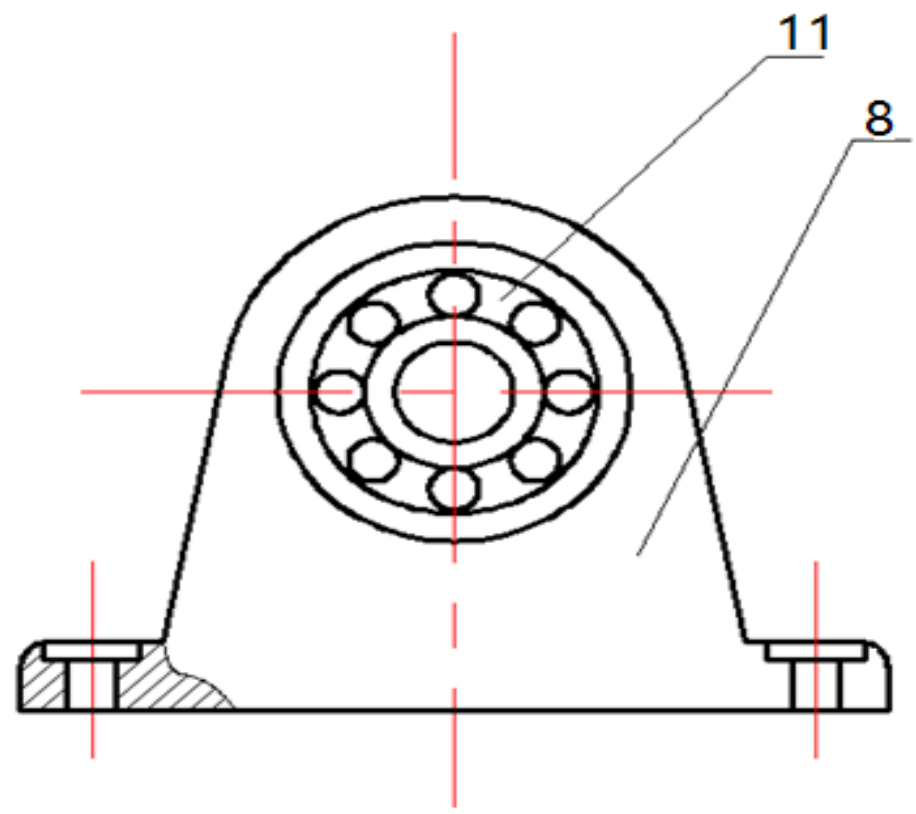

In the picture: 8 - bracket, 11 - angular contact ball bearings

\section{The use of automatic dust-free blackboard eraser process}

Integral part as shown in figure 1 and figure 2, motor 1 through shafts 2 decelerator, 3 drive screw reducer 4 do rotary motion. 6 fixed pulley on the screw, two screw up and down by belt 5 synchronous rotation. Screw 4 fixed on the wall through support 8 and bolt 7 fixed, screw 11with angular contact ball bearing under 4 to bear axial force and radial force. Through screw nut lieutenant rotary motion into linear motion, realize the process of wiping the blackboard. There is a travel switch around 10 near the blackboard end face to ensure safety. The eraser part as shown in figure 3, in the process of an eraser around mobile. Fan motor 12 turns the fan 19 of the eraser will chalk dust sucked into the eraser cavity by 14 to filter dust filter down to wipe clean process. Work process; to wipe a blackboard by press the switch board, when moving the eraser end to the blackboard, the eraser to touch the switch contact when the power disconnect wipe the end of the process. The eraser reverse move when press the switch again, the working process of the same as above. 


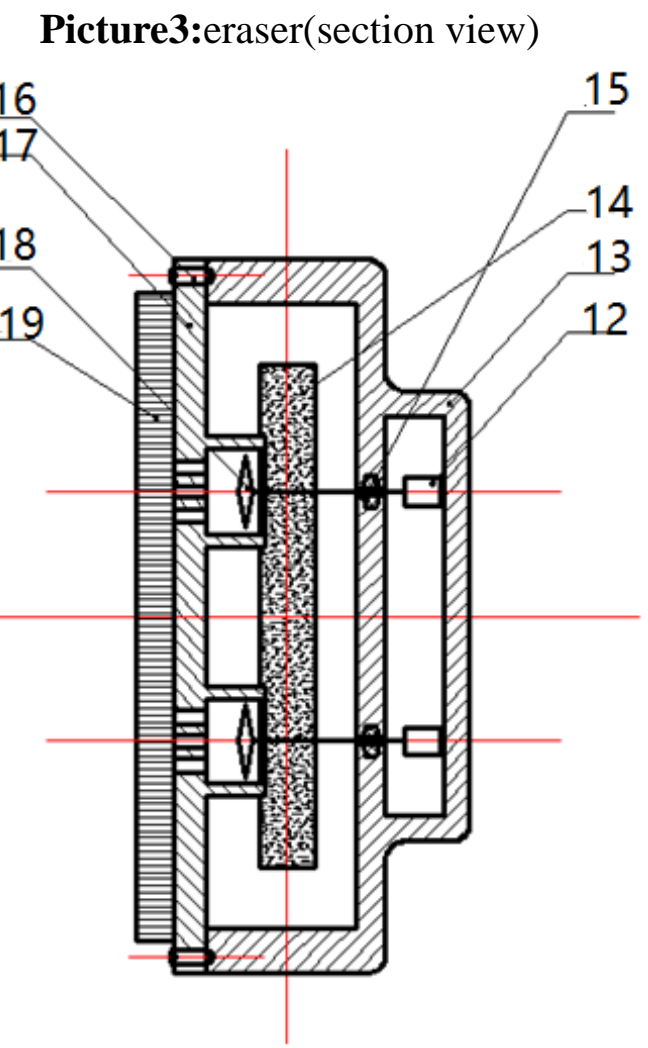

In the picture: 12 - fan motor, 13 - groove after an eraser, 14 - filter, 15 - seal, 16 - end cover screw, 17 - the eraser end cover, 18 - fan, 19 - the blackboard brush

\section{Conclusions.}

(1) This design to replace power supply with motor, to replace human with power, improving the efficiency, saving time and effort.

(2)Clean the function can make people from inhalation of dust, the protection of the body is great, vital to protect the interests of people and respond to the environmental protection policy.

(3) Application prospect. A vast body of teachers and students to the school, automatic dust-free blackboard eraser will have broad prospect, it will let the majority of teachers and students real experience excellent characteristics of automatic dust-free blackboard eraser.

\section{References}

[1]Cao Weiqing, Xu Zhengyin, Mechanism Design, China Machine Press, May in 2000

[2] Zhang Chunlin, Machanical InnovativeDesign, China Machine Press, in 1999

[3] Zhang Youchen Tsinghua University Press,in January 2011

[4] Zhang Meilin Tsinghuaiversity Press, in January 2011

[5]Wang Zhiping, Higher Education Press, in April 2013 